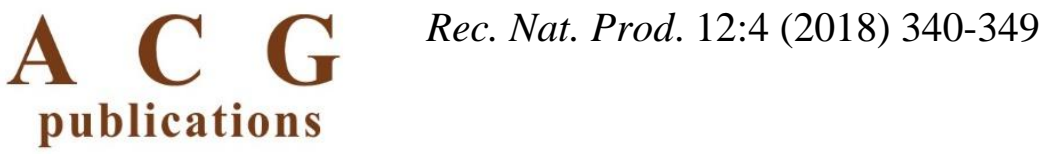

records of natural products

\title{
Chemical Composition and the Potential of
}

\section{Lavandula angustifolia L. Oil as a Skin Depigmentant}

\author{
Felicia Andrei $\odot^{1 *}$, Alexa Ersilia $\odot^{2}$, Camelia Tulcan $\odot^{3}$ and \\ Anca Dragomirescu ${ }^{1}$
}

\author{
${ }^{I}$ Faculty of Farmacy, Department, Dermatopharmacy and Cosmetology Victor Babes University of \\ Medicine and Farmacy, Piata E. Murgu 2, Timisoara 300041, Romania \\ ${ }^{2}$ Faculty of Food Processing, Banat's University of Agricultural Sciences and Veterinary Medicine \\ "King Michael I of Romania" from Timisoara, Calea Aradului 119, Timisoara 300645, Romania \\ ${ }^{3}$ Faculty of Veterinary Medicine,, Banat's University of Agricultural Sciences and Veterinary \\ Medicine "King Michael I of Romania” from Timisoara, Calea Aradului 119, Timisoara 300645, \\ Romania
}

(Received October 03, 2017; Revised November 15, 2017; Accepted November 18, 2017)

\begin{abstract}
Finding non-invasive skin depigmenting agentsrepresent an important goal of cosmetic research and industry. It is now admited that Tyrosinase inhibitor substances could be the most efficient molecules in this field because Tyrosinase is the key enzyme in the melanogenesis process. In the pathology of macular skin hyperpigmentations it can mention aesthetic problems such as: melasma gravidarum, pigmentation of aged skin, photosensibilisation, hepatic disfunctions and post-inflammatory residual pigmentation. The aim of the study was to find out if the Lavandula essential oil has depigmenting properties on human skin, having as theoretical premise its tyrosinase-blocking effect, previously reported in literature for some plant species. By using gas chromatograph coupled with mass spectrometer (GC/MS), a pharmaceutical cream preparation, a protocol of exploration on volunteers (including criteria) and a mexameter, we have highlighted the composition of a sample of Lavandula angustifolia essential oil (LEO) and its depigmenting effect on skin melanic macula. The results highlightes that the main compounds identified were linalool $(26.783 \%)$, terpinen-4-ol (22.143\%) and 3carene(21.668\%), Terpinen-4-ol is represented as the possible active compound in tyrosinase inhibition. Our dermocosmetic assay shows that the depigmenting effect of Lavandula angustifolia oil is effective after 2 months of daily topical treatement, when the melanine average value (measured by the mexameter) decreases more than a third from the innitial value. The study remarks that Lavandula angustifolia essential oil (LEO) in daily cutaneous pharmaceutical form application, during two month, reduces the intensity of spot skin melanin, exprimed through mexametric values.
\end{abstract}

Keywords: Lavandula angustifolia L.; tyrosinase inhibitor; melanic skin macula. (C) 2018 ACG Publications. All rights reserved.

\section{Introduction}

Eliminating or at least reducing hyperpigmentated macules - especially facial located - is a major problem in dermatocosmetics. There are two main reasons for trying to solve this skin condition. The first is aesthetic, continuously promoted by comercial advertising and justified by growing demands on the quality of life. In fact the consumers demand represent the research and

*Corresponding author: E-Mail:andreifelicia@yahoo.com; Phone: +40-726-386-390;+40-256-494-604 
innovation engine forthe pharmaceutical industry. In this regard we can mention the aesthetic problems such as melasma gravidarum, senile spots and postacneic (post-inflammatory) residual pigmentation [1]. The second major motivation belongs to the curative medicine because behind most macules of pigmentation may be hidden a real pathology like (phyto)photosensibilization and hepatic dysfunction (because the Melanocyte-stimulatinghormone is normaly catabolized by the liver) [2].Ongoing research and innovation for finding new natural depigmenting agents has become a necessity, especially now that the use of hydroquinone as a classic cosmetic ingredient is avoided, since it cause skin irritation,ochronosis and it is a compound that may have cancer-causing action [3]. From the pacient point of view, the aesthetic medicine methods (resorcinol or trichloroacetic acid deep peels) are not accepted, for the fear of painfull application and side effects: erythema, risk of infections, social isolation, and ironically, even post-procedure pigmentations.

The reassessment of this skin condition came after the elucidation of the mechanism of melanogenesis, physiologically clarified step by step. Today we can say that depigmentants decreasing the synthesis and intraepidermal deposits of melanin are classified as follows: (1) inhibitors of tyrosinase (key enzyme of melanogenesis), (2) DOPA-oxidase blockers, (3) melanosomes transfer inhibitors, in order to block their phagocytosis in neighboring keratinocytes, (4) epidermal turnover accelerating agents, including keratolitics and peeling substances, and (5) the antioxidant substances inhibiting the oxidation of DOPA-quinone [4].However, when we say melanin depigmentant, we actually understand tyrosinase blockers, because they are the most practically used and in theory they should be the most effective (blocking the first stage in the synthesis of melanin, the start). The pharmaceutical practice still knows many agents employed herein: arbutin, kojic acid, ellagic acid, etc, but the poor efficacy of the products always demand finding new depigmenting agents, more compliant, more active, or substances who increase the efficiency of the first one, used together, synergistically [5].

The Lavandula species are one of the most popular medicinal herbs. Lavanda, enjoys a history of over 2000-2500 years, beginning with Theophrastus (370-285 BC), untill the first monograph 1780,Lavandula volatile oils have been on the market since 1976, to our days when in 2009, it is also conditioned as capsules, an authorized product in Germany, for the anxiety treatment [6]. Recently, there are researches regarding the inhibitory effect of Lavandula angustifolia essential oil (LEO) on fungal tyrosinase. In the fungi, tyrosinase plays a different biological role than in the human body. In plants and fungi, tyrosinase can oxidize a variety of phenolic compounds, leading to the undesirable effect of blackening of fruits and vegetables, causing a degradation both on nutritional quality and on the commercial value of commodities [7]. Because of this, depigmenting agents are highly sought after in agriculture and the food technology industry.

Volatile spike lavender oil (Lavandula spica L.) has been investigated by a team of Italian researchers evaluating the inhibitory activity on fungal tyrosinase. Their composition was evaluated (described) by gas chromatography and mass spectrometry. Both oils have inhibitory effect on fungal tyrosinase in a dose-dependent manner [8].

Extrapolating the encouraging result of Fiocco et al 2011 and 2016 [7,8] and knowing that in the human body, tyrosinase is the key enzyme of melanogenesis, the present study attempts to highlight whether this biochemical property (inhibition of tyrosinase by lavender oil) would also have a beneficial impact on melanogenesis. The result could be encouraging and favorable, because after the worldwide avoidance of hydroquinone (due to its significant toxic potential), the other potential natural blockers of tyrosinase have a mediocre clinical effect, requiring continued and stronger, or synergistic use of those highlighted to date.

The aim of this study was: i) to determine the proximate composition of Lavandula angustifolia flowers ; ii) to obtain and characterize the LEO; iii) to demonstrate, on human subjects, the potential to diminishing the melanin of the skin, at the level of unreliable melanic spots (macules of divese causes) by the mexametry method. 


\section{Materials and Methods}

\subsection{Plant Prelevation and LEO Extraction}

The Lavandula angustifolia (LA) flower was taken in June 2016 from the private culture obtained in the western part of Romania. Species identification has been confirmed by the Department of Herbs and Medicinal Plants, USAMVB Timisoara and a voucher sample has been retained (Herbarium number LA5). The plants were dried at room temperature, grunted with GrindomixRetsch GM 2000 Laboratory Mills, and the oil was extracted by water distillation using the Clevenger Equipment.

\subsection{Plant Material Characterisation}

The characterization of the plant material was achieved by determining the proximate composition using the official AOAC methods (AOAC, 1997): protein (method 954.01); Lipids (920.39); Ash (923.03) and humidity (925.09). Macro and microelements analysis was done using atomic absorption spectrophotometry (SAA).

\subsection{LEO Component Analysis Using GC-MS}

The chemical composition of oil was determined using a Agilent Technology 7820A (Agilent Scientific, USA) gas chromatograph (GC/MS) coupled with MSD 5975 mass spectrometer and equipped with a capillary column with the following characteristics: DB WAX (30 m x $250 \mu \mathrm{m}$ x 0.25 $\mu \mathrm{m})$. The carrier gas was helium at a flow rate of $1 \mathrm{~mL} / \mathrm{min}$. For the separation of compounds, the following program was used: $40^{\circ} \mathrm{C}$ for $1 \mathrm{~min}$, rate of $5^{\circ} \mathrm{C} / \mathrm{min}$ to $210^{\circ} \mathrm{C}$ where was maintained for 5 min. Injector and ion source temperatures were $250^{\circ} \mathrm{C}$ and $150^{\circ} \mathrm{C}$ respectively. The injection volume was $1 \mu \mathrm{L}$ at a ratio of 1:20. The NIST 17 Mass spectral Library database was used to identify volatile compounds. The Linear retention indexes (LRI) werecalculated using n-alkanes C8-C20 and compared with NIST databasereported on DB column.

\subsection{Preparation of a Pharmaceutical form with LEO}

A pharmaceutical product was prepared according to the formulas listed in the table $\mathrm{S} 1$ and the Composition according to INCI (International Nomenclature of Cosmetic Ingredients) in the Table S2 of the Supporting Information.

The quantities of ingredients were separated in 4 phases:

Phase A: The lipidic ingredients were heated in a patented water bath at $70-80^{\circ} \mathrm{C}: 10.0 \mathrm{~g}$ of rice germ oil $3.0 \mathrm{~g}$ of mango butter. Montanov ${ }^{\mathrm{TM}} 68$ was then added in a quantity of $3.0 \mathrm{~g}$. This is a non-ionic, liquid crystal emulsifier based on the HLB system. Liquid crystal emulsifiers create a lamellar or bilayered structure that mimics the lipid bilayers in our stratum corneum, which may help actives or lipids from our lotions penetrate our skin easier. They also assist in reducing transepidermal water loss as the lotions hold more water in contact with the skin for a longer period of time. In the heated oil phase it was included Montanov ${ }^{\mathrm{TM}}$ 68, which does best with a $25 \%$ oil phase and works to emulsify natural oils as well as silicones and esters. Including a fatty alcohol like Cetyl or Cetearyl Alcohol can help increase the liquid crystal formation in the lotion. It is compatible with cationic ingredients, like Honeyquat and Cetrimonium Chloride. And it works well from $\mathrm{pH} 3$ to 12, so it's a good choice for making lotions as diverse as light facial moisturizers with AHAs and bleaching or depilatory creams. Lotions made with Montanov ${ }^{\mathrm{TM}} 68$ are described as being lighter and more moisturizing than those made with non-liquid crystal emulsifiers, like Polawax ${ }^{\mathrm{TM}}$ or Emulsifying Wax $\mathrm{NF}$, this being the main reason for choosing him as an ingredient.The $\mathrm{O} / \mathrm{W}$ emulsifier is versatile and 
can be used for natural or "green" concepts and offers variable consistency ranging from spray to thick creams with a light texture. Appearance: white flakes. Stability: stable between pH 3 - 12. HLB:10. Country of Origin: France, Seppic. Approved by ECOCERT, COSMOS and Natrue, preservative-free, synthetised without solvent.

Phase B: $60.0 \mathrm{~g}$ of water, $2.2 \mathrm{~g}$ of Caolin then $0.5 \mathrm{~g}$ Sanfteen was added, which is a nonionic sugar surfactant from coconut oil and sucrose. It is a tough, whitish mass with a somewhat soapy odor, which tends to become liquid at temperatures above $25^{\circ} \mathrm{C}$. Gentle because of its very good skin compatibility (mild and irritation free) is usually worked as co-surfactant in shampoos, shower gels, foam baths and cleansing formulations. The mucosal tolerance is improved by others, with processed surfactants. At the same time, it has a moisturizing and nourishing effect with high fine foaming ability. Therefore it is particularly recommended for sensitive skin and baby products. The $\mathrm{pH}$ is between 7 and 8 , and the wash-active substance (WAS) is $65 \%$. The cosmetic aditive was ordered from the german producer Spinnrad.

Phase C: $9.8 \mathrm{~g}$ of glycerol $0.5 \mathrm{~g}$ of xanthan

Phase D: $1.0 \mathrm{~g}$ Rokonsal TM BSB-N, Lactic acid (Q. S) and the Essential oil of Lavandulaangustifolia. Rokonsal ${ }^{\mathrm{TM}}$ BSB-N is a combination of Benzoic and Sorbic acids in Benzyl alcohol. The ingredients of RokonsalTM BSB conform to the German BDIH recommendation for Natural Cosmetic Products and the recommendations of the "Council of Europe". Due to the organic acids, RokonsalTM BSB may be classified as a "mild preservative", provided the $\mathrm{pH}$ of the final product is not higher than 5. It is effective against Gram-positive and Gram-negative bacteria, yeasts and moulds at acid $\mathrm{pH}$ values. It is produced by Ashland, advancing skin with science. The company develops and produces a comprehensive line of biofunctionals, fixative and styling polymers, bioadhesives, rheology and conditioning polymers for the Personal Care industry. To help formulators create blockbuster innovations, Ashland focuses on breakthrough chemistries that deliver advanced performance properties in hair care, skin care, sun care and oral care products.

\subsubsection{Method of Preparation}

2.4.1.1. Phase A: The solid butter was weighed, then melted to $75^{\circ} \mathrm{C}$, oil was added and then the pot was withdrawn from the hot plate of the heater.

2.4.1.2. Phase B: In water heated to $75^{\circ} \mathrm{C}$, Sanfteen and kaolin was added and mixed until homogeneous. Then phase B was added in phase A, emulsifing for 1-4 minutes.

2.4.1.3. Phase C: Xanthan is dispersed in glycerine, added to phase A / B, and soon emulsified, then gently mixed while it cools, until homogeneous.

2.4.1.4. Phase D: The preservative emulsion is added gradually, the $\mathrm{pH}$ is checked and adjusted if necessary to $\mathrm{pH} 5$ to 5.4 , gently mixing until well incorporated. Only lavender oil will be added at the end.

The ointment contains an increased percentage of lipophilic components and water. This is why this formulation includes: stabilizers, antioxidants, antimicrobial preservatives, emulgators, surfactants and finally plant extracts. Lavandula angustifoliawas chosen rather then Lavandula $x$ intermedia which can be found and harvested in the western part of Romania, for the chemical composition and antimicrobial properties of the essential oil[9].

In the preparation of the emollient ointment, it should be payed attention at the fact that the heating of the lipophilic components is at a temperature of $70-80^{\circ} \mathrm{C}$. The emulsion of the aqueous phase in the lipophilic phase is recommended at the same temperature. Only the volatile oil was added after cooling, in order to avoid changing its structure with increasing temperature.

Mixing a $\mathrm{O} / \mathrm{W}$ alkylpolyglucoside emulsifier of natural origin, it can be obtained a rich, easyto-spread cream. As a promoter of liquid crystals which biomimic the lipid bilayer of the cellular membrane, it helps to stabilize the emulsion, provides the restructuring effect (reduction of TEWL) and the moisturizing effect. 
The obtained $\mathrm{O} / \mathrm{W}$ emulsion contains a fatty phase of almost $23 \%$ requiring minimum $3 \%$ of emulsifying factors. This creamy white emulsion with lavender smell is extremely easy to apply and non-irritant, even for sensitive, dry and mature skin. It can be appreciated that the lavender oil can be not only a flavouring but an active cosmetic ingredient in a higher concentration[10].

\subsection{The Dermocosmetic Assay}

The mexametric assay was performed on 15 volunteers, all women, aged between 22 and 64 years, which accepted to applied 2 times a day the cream prepared in our laboratory, for a 2-month horizon. The criteria for including the volunteers in the study were: (1) Written consent of the volunteers after they understood the test procedure, (2) Diagnostic of the melanic macula, without a tegumentaryrelief (melasma, spot of photosensibilisation, senile or postacneic (post-inflammatory) residual pigmentations), (3) Lack of any pathological conditions on the explored tegumentary area (eg. acne, seborrheic dermatitis, psoriasis)(4) Elimination of cutaneous intolerance, after a preliminary test of the cream at a topical skin application.

The intensity of the melanin colour (and the possible diminishing of skin coloration) we used the Mexameter MX 18, produced by produced by Courage Khazaka (DE). This is a very simple, fast and economical tool used to measure the two components, mainly responsible for skin color: melanin and hemoglobin (erythema).

The ethics committee of the Faculty of Pharmacy in Timişoara approved the study on volunteers with the mexamethry, the noninvasive method for evaluation of skin coloring (Protocol number 6/22.02.2017).

\subsection{Statistical Analysis}

In order to observe if our results have significances we used the ANOVA one way method.Additionally, layout of the design and statistical analysis is simple. Aframework for one-way ANOVA following a single factor with more data levels has been found to be ideal. Results are presented as means \pm standard deviation (SD) of triplicate measurements. Analysis of variance was performed on the data recorded using statistical data analysis, from EXCEL.

\section{Results and Discussion}

\subsection{Chemical composition}

The proximate composition and the content of macro and microelements of Lavandula angustifolia flowers are presented in table S3 and 1, while the chemical composition of LEO are shown in Table 2.

Table 1. Macro and microelements composition of Lavandula angustifolia*

\begin{tabular}{lllllllll}
\hline $\mathbf{K}$ & $\mathbf{C a}$ & $\mathbf{M g}$ & $\mathbf{N a}$ & $\mathbf{M n}$ & $\mathbf{F e}$ & $\mathbf{Z n}$ & $\mathbf{N i}$ & $\mathbf{C u}$ \\
\hline $3437 \pm 0.62$ & $5000 \pm 0.20$ & $598 \pm 0.15$ & $92.65 \pm 0.50$ & $24.72 \pm 2.25$ & $186 \pm 0.5$ & $4.632 \pm 0.25$ & $0.145 \pm 0.33$ & $0.72 \pm 0.40$ \\
\hline
\end{tabular}

$*$ Results are given in ppm

The values we found in the composition of Lavandula angustifolia are in accord with previously data reported in the literature: $8.71 \%$ moisture, $9.16 \%$ ash, $6.82 \%$ protein, $6.89 \%$ lipids and $61.37 \%$ carbohydrates $[11,12]$. Experimental results on elemental composition reveal that calcium was the most abundant macro element, followed by potassium, magnesium and sodium. Similar values of macro and microelement content in Lavandula angustifolia have been reported previously [13,14].

In Lavandula angustifolia essential oil (LEO) were identified 11 major compounds, in concentration over $0.3 \%$. The main compounds identified were linalool, (26.783\%), terpinen-4-ol (22.143\%) and 3-carene(21.668\%), representing 70.594\% from all analysed compounds (table 2). 
Also, other compounds as: thymol (11.104\%), o-cymene (6.238\%), $\beta$-farnesene (6.587\%), and Lavandulyl acetate $(3.291 \%)$ were found in percentage over $3 \%$ from total.

The presence of compounds belonging to a specific class of secondary metabolites, within a species or even within a single plant may serve as chemotaxonomic markers indicating the biosynthetic pathways and the functional properties of plant [15]. Secondary metabolites are the compounds that usually perform non-essential functions in the plants [16]. Terpenoidsrepresent secondary metabolites found in essential oils of plants widely utilized for chemotaxonomic classification. These groups of compounds exhibit a wide variation in chemical diversity, distribution and function [16].

In the analyzed oil, the monoterpenic oxigenated compounds are predominant $(60.419 \%)$, followed by monoterpenehydrocarbonates $(29.704 \%)$ and sesquiterpene oxygenated (6.587\%) from all chemical compounds.The group of Smigielski et al., 2009 identified a similar distribution of oxigenated monoterpene that represents $73 \%$ of all compounds, hydrocarbonatesmonoterpene $(10.3 \%)$ and oxygenated sesquiterpene (4.8\%) [17].

Table 2. Chemical composition of Lavandula angustifolia essential oil

\begin{tabular}{|c|c|c|c|c|}
\hline No.crt. & Compounds & $\begin{array}{l}\text { LRI reported in } \\
\text { literature* }\end{array}$ & $\begin{array}{l}\text { Liniar Retention } \\
\text { index }(\text { LRI })^{* * *}\end{array}$ & $\%$ \\
\hline 1 & 3-carene & $997-1027$ & 1011 & 21.67 \\
\hline 2 & Linalool & $1074-1098$ & 1086 & 26.78 \\
\hline 3 & Terpinen-4-ol & $1148-1180$ & 1177 & 22.18 \\
\hline 4 & o-cymene & $1009-1076$ & 1197 & 6.24 \\
\hline 5 & Thymol & $1260-1289$ & 1290 & 11.10 \\
\hline 6 & Lavandulyl acetate & $1268-1276$ & 1293 & 3.29 \\
\hline 7 & $\beta$-farnesene & $1428-1458$ & 1449 & 6.59 \\
\hline 8 & $\alpha$-muurolene & $1486-1496$ & 1460 & 0.44 \\
\hline 9 & $\alpha$-caryophyllene & $1396-1419$ & 1462 & 0.99 \\
\hline 10 & Y-muurolene & $1467-1479$ & 1482 & 0.37 \\
\hline \multirow[t]{6}{*}{11} & $\alpha$-bisabolol & $1649-1686$ & 1690 & 0.35 \\
\hline & \multicolumn{3}{|l|}{ Total } & 100 \\
\hline & \multicolumn{3}{|c|}{ Monoterpenehydrocarbonates } & 29.70 \\
\hline & \multicolumn{3}{|c|}{ Sesquiterpene oxygenated } & 6.59 \\
\hline & \multicolumn{3}{|c|}{ Monoterpeneoxigrnated } & 63.71 \\
\hline & \multicolumn{3}{|c|}{ Yield of essential oil \% (w/v) } & 3.4 \\
\hline
\end{tabular}

* Linear retention indices (LRI) according to [15],[16],[18]

** Linear retention indices (LRI) calculated against n-alkanes (C8-C27) for DB WAX

The essential oil's composition is different depending on genetic, environmental and maybe agrotechniques factors [19].Previous studies indicate the linalool and linalyl acetate as major compounds [20-23], in Lavandula angustifolia essential oil. Opposite, L Hui et al., 2010 identified linalyl butyrate as main compound (43.73\%), followed by $\alpha$-Ocymene(25.10\%), Eucalyptol $(7.32 \%)$ and Camphor (3.79\%)[23]. In Lavandula angustifolia essential oilprelevated from west Romaniawere identified caryophyllene (24.12\%) and eucalyptol (15.69\%)[9].

The chemical composition influences the biological properties of the essential oils. Closley related with skin and hair pigmentation in mammals, tyrosinase represents the key enzyme of melanogenesis, and its activity can be influenced by the composition of EOs. Kinetic studies of tyrosinase inhibitory activity of 19 essential oils extracted from medicinal plants revealed the high 
activity of Lavandula angustifolia essential oil[8]. Other studies explain the tyrosinase inhibitory activity in close connection with the chemical composition of the essential oils [24,25].

Fiocco, D. et al., 2011 which constituted our premise, has not yet advanced any explanation on the relationship between chemical structure and antithyrosinase activity, namely, it does not express which substance in the composition of Lavandula angustifolia essential oil inhibits or blocks tyrosinase [7].

Our research, identifying components in theLavandula angustifolia essential oil, attempts to compare them with other similar components for which anti-tyrosinase activity is certain. In support of this reasoning is the study of Korner A. and Pawelek J., 1982 which highlights the fact that volatile oil of Eucalyptus camaldulensis flowers, has tyrosinase inhibitory properties, and therefore antimelanogenic [26].

By comparing the chemical compositions of the two volatile oils, the "suspects circle" of antithyrosinase substances is limited to terpinen-4-ol, found in high concetration in Lavandulaangustrifolia and Eucalyptus camaldulensisoils [27].

The future use of Lavandula angustifolia essential oil as a depigmenting natural product would be a great advantage for the cosmetics industry[28]. Lavanadula is more widely spread than eucalyptus (for example) and essential lavender oil is a patient-friendly product and well received by the consumer.

\subsection{Results Concerning the Dermocosmetic Assay}

The melanine values for the 15 volunteers were calculated as arithmetic average, for 2 cutaneous areas: near (outside) the melanine macula and respectively inside the melanine macula. The melanin concentration inside the stain is higher compared to the outside, reacting more strongly in the positive sense to the applied product. Those results are shown in the table 3.

Table 3.The values of melanine in the melanine macula, during the mexametry assay

\begin{tabular}{l|cc}
\multicolumn{3}{|c}{ Melanine inside vs. outside of the macula } \\
\hline & Outside & Inside \\
\hline 2 & 99.67 & 174.67 \\
3 & 93.32 & 172.30 \\
4 & 92.27 & 170.33 \\
5 & 88.11 & 152.12 \\
6 & 89.15 & 147.27 \\
7 & 89.72 & 132.26 \\
8 & 92.12 & 112.92 \\
$\mathbf{n}$ & 97.25 & 103.71 \\
$\mathbf{X}$ & 8 & 8 \\
$\mathbf{S}$ & 92.50 & 145.41 \\
$\mathbf{X}_{\mathrm{a}}$ & 4.10 & 27.62 \\
\end{tabular}

Literature finds similar results for plant species such as Glycyrrhizaglabra (as a tyrosinase inhibitor), Arctostaphylosuva-ursi containing arbutin and kojic acid from fungal sources, as well as Glycine max extracts as an inhibitor of the melanosomal transfer [29].

Against all of them, Lavandaangustifolia is more geographically spread and more attractive to patients who see Lavanda essential oil, in their subconscious, as a fragrant product. In addition, Soy extracts are controversial because consumers associate them with the idea of a genetically modified 
plant. In fact, the pharmaceutical research does not seek the supremacy of a species at the expense of another, but the identification of several extracts that, by mixing them in a cosmetic product, reducing the concentration of each and avoiding hydroquinone.

Using the software that analyzed all input data, its own statistical analysis table has resulted and is presented in the tabel 4. Detailed, specialized explanations follow it.

Table 4. Statistical analysis of the data

\begin{tabular}{rrcccc}
\hline source & df & SS & MS & F & P-value \\
\hline \multirow{3}{*}{ treatments } & & 11.199 & 14.990 & 287 & 0.01 \\
& 14 & 0.460 & 0.460 & 0.248 & 1.565 \\
error & 1 & 458.42 & 389.88 & & \\
& & 8 & 8 & & \\
total & 15 & 0.888 & & & \\
\hline
\end{tabular}

F distribution have been used in this given situation, depending on the number of degrees of freedom. Once the appropiate F distribution was determined, we rejected the null hypothesis. The total Sum of the Squares (SS) is the sum of the squares of the deviation of individual observations taken toghether and the Mean Square (MS) is due to the treatment. When these calculations have been performed within the group, it was evident that our assumption was true. Also the $\mathrm{P}$ value beingtypically $<0.05$ is significant, meaning that our results indicate a melanin decrease worth of consideration, especially inside the melanine macula

This evolution is presented in the Figure S1 in Supporting information file.

Lavandula angustifolia essential oil was cited in literature for its tyirosinasis inhibitory property[7, 8, 22, 23]. This effect was demonstrated for the botanical world, on fungi. Extrapolating this effect on the human melanine synthesis, and taken in consideration that tyrosinasis is the key enzime in melanogenesis, this study shows very clear that the depigmenting effect is visible, and its decreasing can be measured by mexametry.

In our study, the depigmenting effect was effective only after minimum 2 months of daily treatement, when the melanineaverage value decreases more than a third from the initial value, but close to the natural skin melanine average value.

This diminution of melanine inside the spot is independent of the natural evolution of the healthy skin (tanning, e.g). For the flat evolution for the first 3 weeks, it is suspected that the epidermis keeps the mature melanosomes inside it at least for a keratinocyte turnover (for a cycle cell time). For the epidermis, the cycle cell is about 28 days. After this time horizon, the epidermacquireses chances to diminish the excesive, the pathological melanine amount. In order to optimise this effect, we recomand the association of Lavandula oil with other tyrosinasis inhibitors, but most important with keratolitic agents.

Seeing this new property highlighted in the present study for Lavandula angustifolia essential oil (LEO), as well as the rich composition reported by other authors [30], we consider now that Lavandula angustifolia deserves to be reconsidered from the classic aromoterapic plant status to the pharmacologically and dermocosmetic active species.

\section{Acknowledgments}

Part of this study was supported by a grant of the Romanian National Authority for Scientific Research and Innovation (CNCS/CCCDI-UEFISCDI), project number PN III-P2-2.1.BG-2016-0126, within PNCDI III, contract no. BG-15,acronym PNEA. The chemical parts were performed in the Interdisciplinary Research Platform and Antioxidant Systems Research Laboratory A1c, belonging to Banat University of Agricultural Sciences and Veterinary Medicine „King Michael I of Romania” from Timisoara, Romania. The dermatologic assay was performed in the Department of Dermatofarmacy of the Faculty of Pharmacy, Timisoara. 


\section{Supporting Information}

Supporting information accompanies this paper on http://www.acgpubs.org/RNP

\section{ORCID}

ErsiliaAlexa:0000-0003-4641-7365

Felicia Andrei:0000-0002-5668-3520

Camelia Tulcan: 0000-0003-3096-0232

AncaDragomirescu: 0000-0002-8903-9862

\section{References}

[1] J.Y.Lin, E. David andD.E.Fischer (2007). Melanocyte biology and skin pigmentation, Nature 445, 843850.

[2] A.G. Pandia and I.L. Guevara (2000). Disorders of hyperpigmentation, Derm. Clins.18, 91-98.

[3] W. Zhu and J. Gao (2008). The use of botanical extracts as topical skin-lightening agents for the improvement of skin pigmentation disorders, J. Inv. Derm. Symp. Proceeds. 13, 20-24.

[4] Y. Yamaguchi and V.J. Hearing (2009). Physiological factors that regulate skin pigmentation, Biofactors 35, 193-199.

[5] J.W. Shin and K.C. Park (2011). Current clinical use of depigmenting agents, Derm. Sinica 32, 205210.

[6] E. Basch, I. Foppa, R. Liebowitz, J. Nelson, M. Smith, D. Sollars and C. Ulbricht (2004) Lavender (Lavandula angustifolia Miller), J. Herb. Pharm. 4, 12-14

[7] D. Fiocco, D. Fiorentino, L. Frabboni, S. Benvenuti, G. Orlandini, F. Pellati and A. Gallone (2011). Lavender and peppermint essential oils as effective mushroom tyrosinase inhibitors: A basic study, Flavours Fragr. J. 26, 441-446

[8] D. Fiocco, M. Arciuli, M.P. Arena, S. Benvenuti and A. Gallone (2016). Chemical composition and the anti-melanogenic potential of different essential oils, Flavous. Fragr. J. 31, 255-261

[9] C. Jianu, G. Pop, A.T. Gruia and F.G. Horhat (2013). Chemical composition and antimicrobial activity of essential oils of lavender (Lavandula angustifolia) and lavandin (Lavandula $x$ intermedia) grown in Western Romania, Int. J. Agric. Biol.15, 772-776

[10] D. Sabara and A. Kunicka-Styczyska (2009). Lavender oil-flavouring or active cosmetic ingredient, Food Chem. Biotechnol.73, 33-40

[11] A. Adal, L. S. Sarker, A. D. Lemke and S. S. Mahmoud (2017). Isolation and functional characterization of a methyl jasmonate-responsive 3-carene synthase from Lavandula $\mathrm{x}$ intermedia, Plant Molecular Biol. 93, 641-657.

[12] Z. Ullah, K. M. Baloch; I. B. Baloch and B. Farzana (2013). Proximate and nutrient analysis of selected medicinal plants of tank and South Waziristan area of Pakistan, Middle-East J. Sci. Res.13, 1345-1350

[13] S. Yagi, E. Alia, A.B.D. Rahman, O.M.E. Gihan and M.A.M. Abdelhafeez (2013). Analysis of ten Sudanese medicinal plants using X-ray fluorescence, J. Appl. Ind. Sci. 1, 49-53.

[14] I. Queralt, M. Ovejero, M. L. Carvalho, A. F. Marques and J. M. Labres (2005). Quantitative determination of essential and trace element content of medicinal plants and their infusions by XRF and ICP techniques, X-Ray Spectrom. 34, 213-217.

[15] S. L. Crocketta and N. K. B. (2011). Taxonomy and chemotaxonomy of the genus Hypericum, Robsonb. Med. Aromat. Plant. Sci. Biotechnol. 5, 1-13.

[16] R. Singh (2016). Chemotaxonomy: A tool for plant classification, J. Med. Plants Stud. 4(2), 90-93.

[17] K. Smigielski, A. Raj, K. Krosowiak and R. Gruska (2013). Chemical composition of the essential oil of Lavandula angustifolia cultivated in Poland, JEOP 12(3), 338-347

[18] V. I. Babushok, P. J. Linstrom and I. G. Zenkevich (2011). Retention indices for frequently reported compounds of plant essential oils, J. Phys. Chem. Ref. Data, 40, 1-47.

[19] C.N. Hassiotis, F. Ntana, D.M. Lazari, S. Poulios and K.E. Vlachonasios (2014). Environmental and developmental factors affect essential oil production and quality of Lavandula angustifolia during flowering period, Ind. Crops Prod. 62, 359-366.

[20] C. Da Porto, D. Decorti and I. Kikic (2009). Flavour compounds of Lavandula angustifolia L. to use in food manufacturing: Comparison of three different extraction methods, Food Chem. Toxicol.112, 10721078 .

[21] S.C. Duda, L.A. Marghitas, D. Dezmirean, M. Duda, R. Margaoan and O. Bobis(2015). Changes in major bioactive compounds with antioxidant activity of Agastache foeniculum, Lavandula angustifolia, 
Melissa officinalis and Nepeta cataria: Effect of harvest time and plant species, Ind. Crops Prod. 77, 499-507.

[22] S. Robu, A. C. Aprotosoaie, A. Spac, O. Cioancă, M. Hăncianu and U. Stănescu (2011). Studies regarding chemical composition of lavender volatile oils, Rev Med Chir Soc Med Nat Iasi. 115, 584-589

[23] L. Hui, L. He, L. Huan, X.L Lan and L. A.X.Zhou (2010). Chemical composition of lavender essential oil and its antioxidant activity and inhibition against rhinitis related bacteria, Afr. J. Microb. Res. 4, 309313.

[24] R.S. Verma, L.U. Rahman, C.S. Chanotiya, R.K. Verma, A. Chauhan, A. Yadav, A. Singh and A.K. Yadav (2010). Essential oil composition of Lavandulaa ngustifolia Mill. cultivated in the mid hills of Uttarakhand, India, J. Serbian Chem. Soc. 75, 343-348.

[25] Z. Aumeeruddy-Elalfi, A.Gurib-Fakim and M.F. Mahomoodally (2016). Kinetic studies of tyrosinase inhibitory activity of 19 essential oils extracted from endemic and exotic medicinal plants, S. Afr. J. Bot. 103, 89-94

[26] A. Korner and J. Pawelek (1982). Mammalian tyrosinase catalyzes three reactions in the biosynthesis of melanin, Science 217, 1163-1165

[27] H.C. Huang, Y.C. Ho, J.M. Lim, T.Y. Chang, C.L. Ho and T.M. Chang (2015). Investigation of the anti-melanogenic and antioxidant characteristics of Eucalyptus camaldulensis flower essential oil and determination of its chemical composition, Int. J. Mol. Sci. 16, 10470-10490.

[28] A. Dragomirescu, G. Simu and C. Dehelean (2016). Implementation of mexametry in periorbital hyperpigmentation, Macedonian Pharm. Bull.62, 351 - 352

[29] J.J. Leyden, B. Shergill, G. Micali, J. Downie and W. Wallo (2011). Natural options for the management of hyperpigmentation, J. Eur. Acad. Derm. Vener. 25, 1121-1244

[30] G. Topçu, G. Herrmann, U. Kolak, A.C. Gören, A. Porzel, T.M. Kutchan (2007) Isolation of fatty acids and aromatics from cell suspension cultures of Lavandula angustifolia, Nat. Prod. Res. 21, 100-105

$$
\text { C } 2018 \text { ACG Publications }
$$

